\title{
Impulsive Hilfer fractional differential equations
}

\author{
Hamdy M. Ahmed ${ }^{1 *}$ (D), Mahmoud M. El-Borai ${ }^{2}$, Hassan M. El-Owaidy ${ }^{3}$ and Ahmed S. Ghanem ${ }^{1}$
}

${ }^{*}$ Correspondence:

hamdy_17eg@yahoo.com

${ }^{1}$ Higher Institute of Engineering, El-Shorouk Academy, Cairo, Egypt

Full list of author information is

available at the end of the article

\begin{abstract}
Existence and controllability results for nonlinear Hilfer fractional differential equations are studied. Sufficient conditions for existence and approximate controllability for Sobolev-type impulsive fractional differential equations are established, where the time fractional derivative is the Hilfer derivative. An example for Sobolev-type Hilfer fractional delay partial differential equation with impulsive condition is considered.
\end{abstract}

MSC: 93B05; 26A33; 34A37; 34K37

Keywords: Impulsive condition; Sobolev-type Hilfer fractional differential equation; Controllability; Approximate controllability; Contraction mapping principle

\section{Introduction}

Nonlinear fractional differential equations can be observed in many areas such as population dynamics, heat conduction in materials with memory, seepage flow in porous media, autonomous mobile robots, fluid dynamics, traffic models, electro magnetic, aeronautics, economics, and so on [1-10]. Controllability results for linear and nonlinear integer order differential systems were studied by several authors (see [11-21]). In Sect. 2, we shall present some basic definitions and lemmas concerning fractional calculus. In Sect. 3, we shall study the existence and controllability results for nonlinear Hilfer fractional differential equations. In Sect. 4, we shall investigate the sufficient conditions for existence and approximate controllability for Sobolev-type impulsive fractional differential equations. In Sect. 5, we consider an example for Sobolev-type Hilfer fractional delay partial differential equation with impulsive condition.

\section{Preliminaries}

In order to study the existence, controllability, and approximate controllability for delay Hilfer fractional differential equations with impulsive condition, we need the following basic definitions and lemmas.

Definition 2.1 (see [22]) The fractional integral operator of order $\mu>0$ for a function $f$ can be defined as

$$
I^{\mu} f(t)=\frac{1}{\Gamma(\mu)} \int_{0}^{t} \frac{f(s)}{(t-s)^{1-\mu}} d s, \quad t>0,
$$

where $\Gamma(\cdot)$ is the gamma function.

(c) The Author(s) 2018. This article is distributed under the terms of the Creative Commons Attribution 4.0 International License (http://creativecommons.org/licenses/by/4.0/), which permits unrestricted use, distribution, and reproduction in any medium, provided you give appropriate credit to the original author(s) and the source, provide a link to the Creative Commons license, and indicate if changes were made. 
Definition 2.2 (see $[23,24]$ ) The Hilfer fractional derivative of order $0 \leq v \leq 1$ and $0<\mu<1$ is defined as

$$
D_{0+}^{v, \mu} f(t)=I_{0+}^{v(1-\mu)} \frac{d}{d t} I_{0+}^{(1-v)(1-\mu)} f(t) .
$$

Through this paper, let $E$ be a Banach space with $\|\cdot\|$, and let $P C(J, E)$ be the Banach space of all continuous maps from $J=(0, b]$ into $E$.

Define $Y=\left\{x: t^{(1-v)(1-\mu)} x(t) \in P C(J, E)\right\}$, with the norm $\|\cdot\|_{Y}$ defined by $\|\cdot\|_{Y}=$ $\sup _{t \in J}\left\|t^{(1-v)(1-\mu)} x(t)\right\|$. Obviously, $Y$ is a Banach space.

Introduce the set $B_{r}=\left\{x \in Y:\|x\|_{Y} \leq r\right\}$, where $r>0$.

For $x \in E$, we define two families of operators $\left\{S_{v, \mu}(t): t>0\right\}$ and $\left\{P_{\mu}(t): t>0\right\}$ by

$$
\begin{aligned}
& S_{v, \mu}(t)=I_{0_{+}}^{\nu(1-\mu)} P_{\mu}(t), \quad P_{\mu}(t)=t^{\mu-1} T_{\mu}(t), \\
& T_{\mu}(t)=\int_{0}^{\infty} \mu \theta \Psi_{\mu}(\theta) S\left(t^{\mu} \theta\right) d \theta,
\end{aligned}
$$

where

$$
\Psi_{\mu}(\theta)=\sum_{n=1}^{\infty} \frac{(-\theta)^{n-1}}{(n-1) ! \Gamma(1-n \mu)}, \quad 0<\mu<1, \theta \in(0, \infty)
$$

is a function of Wright type which satisfies

$$
\int_{0}^{\infty} \theta^{\tau} \Psi_{\mu}(\theta) d \theta=\frac{\Gamma(1+\tau)}{\Gamma(1+\mu \tau)}
$$

for $\theta \geq 0$.

Lemma 2.1 (see [25]) The operators $S_{v, \mu}$ and $P_{\mu}$ have the following properties.

(i) $\left\{P_{\mu}(t): t>0\right\}$ is continuous in the uniform operator topology.

(ii) For any fixed $t>0, S_{v, \mu}(t)$ and $P_{\mu}(t)$ are linear and bounded operators, and

$$
\left\|P_{\mu}(t) x\right\| \leq \frac{M t^{\mu-1}}{\Gamma(\mu)}\|x\|, \quad\left\|S_{v, \mu}(t) x\right\| \leq \frac{M t^{(\nu-1)(1-\mu)}}{\Gamma(v(1-\mu)+\mu)}\|x\| .
$$

(iii) $\left\{P_{\mu}(t): t>0\right\}$ and $\left\{S_{v, \mu}(t): t>0\right\}$ are strongly continuous.

\section{Existence and controllability results}

We consider the following nonlinear delay Hilfer fractional differential equation with impulsive condition of the form

$$
\left\{\begin{array}{l}
D_{0+}^{v, \mu} x(t)=A x(t)+f\left(t, x\left(\gamma_{1}(t)\right), \int_{0}^{t} h(t, s) g\left(s, x\left(\gamma_{2}(s)\right)\right) d s\right), \\
\quad t \in J=(0, b], t \neq t_{k}, \\
\left.\Delta x\right|_{t=t_{k}=} I_{k}\left(x\left(t_{k}^{-}\right)\right), \quad k=1,2, \ldots, m \\
I_{0+}^{(1-v)(1-\mu)} x(0)=x_{0}
\end{array}\right.
$$

where $D_{0+}^{v, \mu}$ is the Hilfer fractional derivative, $0 \leq v \leq 1,0<\mu<1, A$ is a closed, linear, and densely defined operator in $E$. The delay $\gamma_{i}(t): J \rightarrow J, i=1,2$, are continuous functions, the 
state $x(\cdot)$ takes values in the Banach space $E$, and $h: J \times J \rightarrow R$ is a continuous function, $\left.\Delta x\right|_{t=t_{k}}=I_{k}\left(x\left(t_{k}^{-}\right)\right)$, where $x\left(t_{k}^{+}\right)$and $x\left(t_{k}^{-}\right)$represent the right and left limits of $x(t)$ at $t=t_{k}$, respectively, and the nonlinear operators $f: J \times E \times E \rightarrow E, g: J \times E \rightarrow E$ are given. The operator $A$ is the infinitesimal generator of a $C_{0}$-semigroup $T(t)$ on $E$, and there exists a constant $M>0$ such that $\|T(t)\| \leq M$.

To establish the results, we need the following hypotheses.

(H1) (i) $f: J \times E \times E \rightarrow E$ is continuous and there exist constants $N_{1}>0$ and $N_{2}>0$ such that, for all $t \in J, v_{1}, v_{2}, w_{1}, w_{2} \in E$, we have

$$
\left\|f\left(t, v_{1}, w_{1}\right)-f\left(t, v_{2}, w_{2}\right)\right\| \leq N_{1}\left[\left\|v_{1}-v_{2}\right\|+\left\|w_{1}-w_{2}\right\|\right], \quad N_{2}=\|f(t, 0,0)\| .
$$

(ii) $g: J \times E \rightarrow E$ is continuous and there exist constants $L_{1}>0$ and $L_{2}>0$ such that, for all $t \in J, v_{1}, v_{2} \in E$, we have

$$
\left\|g\left(t, v_{1}\right)-g\left(t, v_{2}\right)\right\| \leq L_{1}\left\|v_{1}-v_{2}\right\|, \quad L_{2}=\|g(t, 0)\| .
$$

(iii) The functions $I_{k}: E \rightarrow E$ are continuous and there exist constants $L_{3}>0, L_{4}>0$ such that, for all $t \in J, v_{1}, v_{2} \in E$, we have

$$
\left\|I_{k}\left(v_{1}\right)-I_{k}\left(v_{2}\right)\right\| \leq L_{3}\left\|v_{1}-v_{2}\right\|, \quad L_{4}=\left\|I_{k}(0)\right\|, t \in J .
$$

(H2) There exists a constant $L$ such that $|h(t, s)| \leq L$ for $(t, s) \in J \times J$.

(H3) There exists a constant $q$ such that, for all $x_{1}, x_{2} \in E,\left\|x_{1}\left(\gamma_{i}(t)\right)-x_{2}\left(\gamma_{i}(t)\right)\right\| \leq$ $q\left\|x_{1}(t)-x_{2}(t)\right\|$ for $i=1,2$.

(H4) There exists a constant $r>0$ such that

$$
\begin{aligned}
& \left\{\frac{3 M}{\Gamma(v(1-\mu)+\mu)}\left[\left\|x_{0}\right\|+m\left(L_{3} r+L_{4}\right)\right]\right. \\
& \left.\quad+\frac{3 M b^{\mu+(1-v)(1-\mu)}}{\Gamma(\mu+1)}\left[N_{1}\left(r+b L\left(L_{1} r+L_{2}\right)\right)+N_{2}\right]\right\} \leq r
\end{aligned}
$$

and

$$
\begin{aligned}
& \left\{\frac{4 M}{\Gamma(v(1-\mu)+\mu)}\left[\left\|x_{0}\right\|+m\left(L_{3} r+L_{4}\right)\right]+\frac{4 M b^{1-v(1-\mu)}}{\Gamma(\mu+1)}\left[N_{1}\left(r+b L\left(L_{1} r+L_{2}\right)\right)+N_{2}\right]\right\} \\
& \quad \times\left[1+\frac{M b^{\mu}\|B\|\left\|W^{-1}\right\|}{\Gamma(\mu+1)}\right]+\frac{4 M b^{1-v(1-\mu)}\left\|x_{1}\right\|\|B\|\left\|W^{-1}\right\|}{\Gamma(\mu+1)} \leq r .
\end{aligned}
$$

Definition 3.1 (see [25]) We say that $x \in Y$ is a mild solution of equation (3.1) if it satisfies

$$
\begin{aligned}
x(t)= & S_{v, \mu}(t) x_{0}+\int_{0}^{t} P_{\mu}(t-s) f\left(s, x\left(\gamma_{1}(s)\right), \int_{0}^{s} h(s, \tau) g\left(\tau, x\left(\gamma_{2}(\tau)\right)\right) d \tau\right) d s \\
& +\sum_{0<t_{k}<t} S_{v, \mu}\left(t-t_{k}\right) I_{k}\left(x\left(t_{k}^{-}\right)\right), \quad t \in J .
\end{aligned}
$$


Theorem 3.1 If hypotheses (H1)-(H4) are satisfied, then equation (3.1) has a unique mild solution on J provided that

$$
\chi_{1}:=\left\{\frac{3 q b^{\mu} M N_{1}}{\Gamma(\mu+1)}\left[1+b L L_{1}\right]+\frac{3 m M b^{(\nu-1)(1-\mu)}}{\Gamma(v(1-\mu)+\mu)}\right\}<1 .
$$

Proof Consider the operator $\Phi$ on $Y$ defined as follows:

$$
\begin{aligned}
\Phi x(t)= & S_{v, \mu}(t) x_{0}+\int_{0}^{t} P_{\mu}(t-s) f\left(s, x\left(\gamma_{1}(s)\right), \int_{0}^{s} k(s, \tau) g\left(\tau, x\left(\gamma_{2}(\tau)\right)\right) d \tau\right) d s \\
& +\sum_{0<t_{k}<t} S_{v, \mu}\left(t-t_{k}\right) I_{k}\left(x\left(t_{k}^{-}\right)\right) .
\end{aligned}
$$

It will be shown that the operator $\Phi$ has a fixed point.

First we show that $\Phi$ maps $B_{r}$ into itself. For $x \in B_{r}$,

$$
\begin{aligned}
\|\Phi x\|_{Y}= & \sup _{t \in J} t^{(1-\nu)(1-\mu)}\|(\Phi x)(t)\| \\
\leq & 3 \sup _{t \in J} t^{(1-v)(1-\mu)}\left\{\left\|S_{v, \mu}(t) x_{0}\right\|\right. \\
& +\int_{0}^{t}\left(\left\|P_{\mu}(t-s)\right\|\left\|f\left(s, x\left(\gamma_{1}(s)\right), \int_{0}^{s} h(s, \tau) g\left(\tau, x\left(\gamma_{2}(\tau)\right)\right) d \tau\right)\right\| d s\right. \\
& \left.+\sum_{0<t_{k}<t}\left\|S_{v, \mu}\left(t-t_{k}\right) I_{k}\left(x\left(t_{k}^{-}\right)\right)\right\|\right\} \\
\leq & \frac{3 M}{\Gamma(v(1-\mu)+\mu)}\left[\left\|x_{0}\right\|+m\left(L_{3} r+L_{4}\right)\right] \\
& +\frac{3 M b^{\mu+(1-v)(1-\mu)}}{\Gamma(\mu+1)}\left[N_{1}\left(r+b L\left(L_{1} r+L_{2}\right)\right)+N_{2}\right] \\
\leq & r .
\end{aligned}
$$

Thus $\Phi$ maps $B_{r}$ into itself.

We show that $(\Phi x)(t)$ is continuous on $J$ for any $x \in B_{r}$. Let $0<t \leq b$ and $\epsilon>0$ be sufficiently small, then

$$
\begin{aligned}
& \|(\Phi x)(\cdot+\epsilon)-(\Phi x)(\cdot)\|_{Y} \\
& =\sup _{t \in J} t^{(1-v)(1-\mu)}\|(\Phi x)(t+\epsilon)-(\Phi x)(t)\| \\
& \leq 3 \sup _{t \in J} t^{(1-v)(1-\mu)}\left\|\left(S_{v, \mu}(t+\epsilon)-S_{v, \mu}(t)\right) x_{0}\right\| \\
& \quad+3 \sup _{t \in J} t^{(1-v)(1-\mu)} \| \int_{0}^{t+\epsilon} P_{\mu}(t+\epsilon-s) f\left(s, x\left(\gamma_{1}(s)\right),\right. \\
& \left.\quad \int_{0}^{s} h(s, \tau) g\left(\tau, x\left(\gamma_{2}(\tau)\right)\right) d \tau\right) d s \\
& \quad-\int_{0}^{t} P_{\mu}(t-s) f\left(s, x\left(\gamma_{1}(s)\right), \int_{0}^{s} h(s, \tau) g\left(\tau, x\left(\gamma_{2}(\tau)\right)\right) d \tau\right) d s \|
\end{aligned}
$$




$$
\begin{aligned}
& +3 \sup _{t \in J} t^{(1-v)(1-\mu)} \| \sum_{0<t_{k}<t+\epsilon} S_{v, \mu}\left(t+\epsilon-t_{k}\right) I_{k}\left(x\left(t_{k}^{-}\right)\right) \\
& -\sum_{0<t_{k}<t} S_{v, \mu}\left(t-t_{k}\right) I_{k}\left(x\left(t_{k}^{-}\right)\right) \|
\end{aligned}
$$

Clearly, the right-hand side of (3.3) tends to zero as $\epsilon \rightarrow 0$. Hence, $(\Phi x)(t)$ is continuous on $J$.

We are going to show that $(\Phi x)(t)$ is a contraction on $B_{r}$.

Next, for $x_{1}, x_{2} \in B_{r}$, we obtain

$$
\begin{aligned}
\|( & \left(x_{1}\right)(t)-\left(\Phi x_{2}\right)(t) \| \\
\leq & 3 \int_{0}^{t}\left\|P_{\mu}(t-s)\right\| \| f\left(s, x_{1}\left(\gamma_{1}(s)\right), \int_{0}^{s} h(s, \tau) g\left(\tau, x_{1}\left(\gamma_{2}(\tau)\right)\right) d \tau\right) \\
& -f\left(s, x_{2}\left(\gamma_{1}(s)\right), \int_{0}^{s} h(s, \tau) g\left(\tau, x_{2}\left(\gamma_{2}(\tau)\right)\right) d \tau\right) \| d s \\
& +3 \sum_{0<t_{k}<t}\left\|S_{v, \mu}\left(t-t_{k}\right)\right\|\left\|I_{k}\left(x_{1}\left(t_{k}^{-}\right)\right)-I_{k}\left(x_{2}\left(t_{k}^{-}\right)\right)\right\| \\
\leq & \left\{\frac{3 q b^{\mu} M N_{1}}{\Gamma(\mu+1)}\left[1+b L L_{1}\right]+\frac{3 m M b^{(v-1)(1-\mu)}}{\Gamma(v(1-\mu)+\mu)}\right\}\left\|x_{1}(t)-x_{2}(t)\right\| \\
\leq & \chi_{1}\left\|x_{1}(t)-x_{2}(t)\right\| .
\end{aligned}
$$

Therefore,

$$
\sup _{t \in J} t^{(1-v)(1-\mu)}\left\|\left(\Phi x_{1}\right)(t)-\left(\Phi x_{2}\right)(t)\right\| \leq \chi_{1} \sup _{t \in J} t^{(1-v)(1-\mu)}\left\|x_{1}(t)-x_{2}(t)\right\| .
$$

This implies that

$$
\left\|\Phi x_{1}-\Phi x_{2}\right\|_{Y} \leq \chi_{1}\left\|x_{1}-x_{2}\right\|_{Y}
$$

Then, $\Phi$ is a contraction mapping on $B_{r}$. From the Banach fixed point theorem, $\Phi$ has a unique fixed point $x(t)$ on $J$. Therefore system (3.1) has a unique mild solution on $J$, and the proof is completed.

Next, we will establish a set of sufficient conditions for controllability of impulsive delay Hilfer fractional differential equation in the following form:

$$
\left\{\begin{array}{l}
D_{0+}^{v, \mu} x(t)=A x(t)+B u(t)+f\left(t, x\left(\gamma_{1}(t)\right), \int_{0}^{t} h(t, s) g\left(s, x\left(\gamma_{2}(s)\right)\right) d s\right) \\
\quad t \in J=(0, b], t \neq t_{k} \\
\left.\Delta x\right|_{t=t_{k}}=I_{k}\left(x\left(t_{k}^{-}\right)\right), \quad k=1,2, \ldots, m \\
I_{0+}^{(1-v)(1-\mu)} x(0)=x_{0}
\end{array}\right.
$$

where the control function $u(\cdot)$ is given in $L^{2}(J, U)$, the Banach space of admissible control functions with $U$ a Banach space. The symbol $B$ stands for a bounded linear from $U$ into $E$. 
Definition 3.2 We say that $x \in Y$ is a mild solution of system (3.4) if it satisfies

$$
\begin{aligned}
x(t)= & S_{v, \mu}(t) x_{0}+\int_{0}^{t} P_{\mu}(t-s) B u(s) d s \\
& +\int_{0}^{t} P_{\mu}(t-s) f\left(s, x\left(\gamma_{1}(s)\right), \int_{0}^{s} h(s, \tau) g\left(\tau, x\left(\gamma_{2}(\tau)\right)\right) d \tau\right) d s \\
& +\sum_{0<t_{k}<t} S_{v, \mu}\left(t-t_{k}\right) I_{k}\left(x\left(t_{k}^{-}\right)\right), \quad t \in J .
\end{aligned}
$$

Definition 3.3 System (3.4) is said to be controllable on $J$ if, for every $x_{0}, x_{1} \in E$, there exists a control $u \in L^{2}(J, U)$ such that the mild solution $x(t)$ of system (3.4) satisfies $x(b)=$ $x_{1}$, where $x_{1}$ and $b$ are the preassigned terminal state and time, respectively.

To establish the result, we need the following additional hypothesis:

(H5) The linear operator $W$ from $U$ into $E$ defined by

$$
W u=\int_{0}^{b} P_{\mu}(b-s) B u(s) d s
$$

has an inverse operator $W^{-1}$ which takes values in $L^{2}(J, U) \backslash$ ker $W$, where the kernel space of $W$ is defined by $\operatorname{ker} W=\left\{x \in L^{2}(J, U): W x=0\right\}$ and $B$ is a bounded operator.

Theorem 3.2 If hypotheses (H1)-(H5) are satisfied and if

$$
\chi_{2}:=\left\{\frac{4 q b^{\mu} M N_{1}}{\Gamma(\mu+1)}\left[1+b L L_{1}\right]+\frac{4 m M b^{(\nu-1)(1-\mu)}}{\Gamma(\nu(1-\mu)+\mu)}\right\}\left\{1+\frac{M\|B\|\left\|W^{-1}\right\| b^{\mu}}{\Gamma(\mu+1)}\right\}<1
$$

then system (3.4) is controllable on J.

Proof Using assumption (H5), define the control

$$
\begin{aligned}
u(t)= & W^{-1}\left\{x_{1}-S_{v, \mu}(b) x_{0}\right. \\
& -\int_{0}^{b} P_{\mu}(b-s) f\left(s, x\left(\gamma_{1}(s)\right), \int_{0}^{s} h(s, \tau) g\left(\tau, x\left(\gamma_{2}(\tau)\right)\right) d \tau\right) d s \\
& \left.-\sum_{0<t_{k}<b} S_{v, \mu}\left(b-t_{k}\right) I_{k}\left(x\left(t_{k}^{-}\right)\right)\right\}(t) .
\end{aligned}
$$

It shall now be shown that when using this control, the operator $\Phi^{*}$ defined by

$$
\begin{aligned}
\left(\Phi^{*} x\right)(t)= & S_{v, \mu}(t) x_{0}+\int_{0}^{t} P_{\mu}(t-s) f\left(s, x\left(\gamma_{1}(s)\right), \int_{0}^{s} k(s, \tau) g\left(\tau, x\left(\gamma_{2}(\tau)\right)\right) d \tau\right) d s \\
& +\sum_{0<t_{k}<t} S_{v, \mu}\left(t-t_{k}\right) I_{k}\left(x\left(t_{k}^{-}\right)\right) \\
& +\int_{0}^{t} P_{\mu}(t-\eta) B W^{-1}\left\{x_{1}-S_{v, \mu}(b) x_{0}\right. \\
& -\int_{0}^{b} P_{\mu}(b-s) f\left(s, x\left(\gamma_{1}(s)\right), \int_{0}^{s} h(s, \tau) g\left(\tau, x\left(\gamma_{2}(\tau)\right)\right) d \tau\right) d s
\end{aligned}
$$




$$
\left.-\sum_{0<t_{k}<b} S_{v, \mu}\left(b-t_{k}\right) I_{k}\left(x\left(t_{k}^{-}\right)\right)\right\}(\eta) d \eta
$$

has a fixed point. This fixed point is then a solution of equation (3.4).

First we show that $\Phi^{*}$ maps $B_{r}$ into itself. For $x \in B_{r}$,

$$
\begin{aligned}
& \left\|\Phi^{*} x\right\|_{Y}=\sup _{t \in J} t^{(1-v)(1-\mu)}\left\|\left(\Phi^{*} x\right)(t)\right\| \\
& \leq 4 \sup _{t \in J} t^{(1-v)(1-\mu)}\left\{\left\|S_{v, \mu}(t) x_{0}\right\|+\int_{0}^{t}\left\|P_{\mu}(t-\eta)\right\|\|B\|\left\|W^{-1}\right\| \| x_{1}-S_{\nu, \mu}(b) x_{0}\right. \\
& -\int_{0}^{b} P_{\mu}(b-s) f\left(s, x\left(\gamma_{1}(s)\right), \int_{0}^{s} h(s, \tau) g\left(\tau, x\left(\gamma_{2}(\tau)\right)\right) d \tau\right) d s \\
& -\sum_{0<t_{k}<b} S_{v, \mu}\left(b-t_{k}\right) I_{k}\left(x\left(t_{k}^{-}\right)\right) \|(\eta) d \eta \\
& +\int_{0}^{t}\left(\left\|P_{\mu}(t-s)\right\|\left\|f\left(s, x\left(\gamma_{1}(s)\right), \int_{0}^{s} h(s, \tau) g\left(\tau, x\left(\gamma_{2}(\tau)\right)\right) d \tau\right)\right\| d s\right. \\
& \left.+\sum_{0<t_{k}<t}\left\|S_{v, \mu}\left(t-t_{k}\right) I_{k}\left(x\left(t_{k}^{-}\right)\right)\right\|\right\} \\
& \leq \frac{4 M}{\Gamma(\nu(1-\mu)+\mu)}\left[\left\|x_{0}\right\|+m\left(L_{3} r+L_{4}\right)\right]+\frac{4 M b^{\mu}\|B\|\left\|W^{-1}\right\|}{\Gamma(\mu+1)}\left\{b^{(1-v)(1-\mu)}\left\|x_{1}\right\|\right. \\
& +\frac{M}{\Gamma(\nu(1-\mu)+\mu)}\left[\left\|x_{0}\right\|+m\left(L_{3} r+L_{4}\right)\right] \\
& \left.+\frac{M b^{1-v(1-\mu)}}{\Gamma(\mu+1)}\left[N_{1}\left(r+b L\left(L_{1} r+L_{2}\right)\right)+N_{2}\right]\right\} \\
& +\frac{4 M b^{1-v(1-\mu)}}{\Gamma(\mu+1)}\left(N_{1}\left(r+b L\left(L_{1} r+L_{2}\right)\right)+N_{2}\right) \\
& =\left\{\frac{4 M}{\Gamma(v(1-\mu)+\mu)}\left[\left\|x_{0}\right\|+m\left(L_{3} r+L_{4}\right)\right]\right. \\
& \left.+\frac{4 M b^{1-v(1-\mu)}}{\Gamma(\mu+1)}\left[N_{1}\left(r+b L\left(L_{1} r+L_{2}\right)\right)+N_{2}\right]\right\} \\
& \times\left[1+\frac{M b^{\mu}\|B\|\left\|W^{-1}\right\|}{\Gamma(\mu+1)}\right]+\frac{4 M b^{1-\nu(1-\mu)}\left\|x_{1}\right\|\|B\|\left\|W^{-1}\right\|}{\Gamma(\mu+1)} \leq r .
\end{aligned}
$$

Thus $\Phi^{*}$ maps $B_{r}$ into itself.

We show that $\left(\Phi^{*} x\right)(t)$ is continuous on $J$ for any $x \in B_{r}$. Let $0<t \leq b$ and $\epsilon>0$ be sufficiently small, then

$$
\begin{aligned}
& \left\|\left(\Phi^{*} x\right)(\cdot+\epsilon)-\left(\Phi^{*} x\right)(\cdot)\right\|_{Y} \\
& =\sup _{t \in J} t^{(1-v)(1-\mu)}\left\|\left(\Phi^{*} x\right)(t+\epsilon)-\left(\Phi^{*} x\right)(t)\right\| \\
& \leq 4 \sup _{t \in J} t^{(1-v)(1-\mu)}\left\|\left(S_{v, \mu}(t+\epsilon)-S_{v, \mu}(t)\right) x_{0}\right\| \\
& \quad+4 \sup _{t \in J} t^{(1-v)(1-\mu)}\left\|\int_{0}^{t+\epsilon} P_{\mu}(t+\epsilon-s) B u(s) d s-\int_{0}^{t} P_{\mu}(t-s) B u(s) d s\right\|
\end{aligned}
$$




$$
\begin{aligned}
& +4 \sup _{t \in J} t^{(1-\nu)(1-\mu)} \| \int_{0}^{t+\epsilon} P_{\mu}(t+\epsilon-s) f\left(s, x\left(\gamma_{1}(s)\right), \int_{0}^{s} h(s, \tau) g\left(\tau, x\left(\gamma_{2}(\tau)\right)\right) d \tau\right) d s \\
& -\int_{0}^{t} P_{\mu}(t-s) f\left(s, x\left(\gamma_{1}(s)\right), \int_{0}^{s} h(s, \tau) g\left(\tau, x\left(\gamma_{2}(\tau)\right)\right) d \tau\right) d s \| \\
& +4 \sup _{t \in J} t^{(1-v)(1-\mu)} \| \sum_{0<t_{k}<t+\epsilon} S_{v, \mu}\left(t+\epsilon-t_{k}\right) I_{k}\left(x\left(t_{k}^{-}\right)\right) \\
& -\sum_{0<t_{k}<t} S_{v, \mu}\left(t-t_{k}\right) I_{k}\left(x\left(t_{k}^{-}\right)\right) \| .
\end{aligned}
$$

Clearly, the right-hand side of (3.7) tends to zero as $\epsilon \rightarrow 0$. Hence, $\left(\Phi^{*} x\right)(t)$ is continuous on $J$.

Next, for $x, y \in B_{r}$, we obtain

$$
\begin{aligned}
&\left\|\left(\Phi^{*} x\right)(t)-\left(\Phi^{*} y\right)(t)\right\| \\
& \leq 4 \int_{0}^{t}\left\|P_{\mu}(t-\eta)\right\|\|B\|\left\|W^{-1}\right\|\left[\int_{0}^{b}\left\|P_{\mu}(b-s)\right\|\right. \\
& \times \| f\left(s, x\left(\gamma_{1}(s)\right), \int_{0}^{s} h(s, \tau) g\left(\tau, x\left(\gamma_{2}(\tau)\right)\right) d \tau\right) \\
&-f\left(s, y\left(\gamma_{1}(s)\right), \int_{0}^{s} h(s, \tau) g\left(\tau, y\left(\gamma_{2}(\tau)\right)\right) d \tau\right) \| d s \\
&\left.+\sum_{0<t_{k}<b}\left\|S_{v, \mu}\left(b-t_{k}\right)\right\|\left\|I_{k}\left(x\left(t_{k}^{-}\right)\right)-I_{k}\left(y\left(t_{k}^{-}\right)\right)\right\|_{Y}\right] d \eta+4 \int_{0}^{t}\left\|P_{\mu}(t-s)\right\| \\
& \times \| f\left(s, x\left(\gamma_{1}(s)\right), \int_{0}^{s} h(s, \tau) g\left(\tau, x\left(\gamma_{2}(\tau)\right)\right) d \tau\right) \\
&-f\left(s, y\left(\gamma_{1}(s)\right), \int_{0}^{s} h(s, \tau) g\left(\tau, y\left(\gamma_{2}(\tau)\right)\right) d \tau\right) \| d s \\
&+4 \sum_{0<t_{k}<t}\left\|S_{v, \mu}\left(t-t_{k}\right)\right\|\left\|I_{k}\left(x\left(t_{k}^{-}\right)\right)-I_{k}\left(y\left(t_{k}^{-}\right)\right)\right\| \\
& \leq \frac{4 M\|B\|\left\|W^{-1}\right\| b^{\mu}}{\Gamma(\mu+1)}\left\{\frac{q b^{\mu} M N_{1}}{\Gamma(\mu+1)}\left[1+b L L_{1}\right]+\frac{m M b^{(\nu-1)(1-\mu)}}{\Gamma(v(1-\mu)+\mu)}\right\}\|x(t)-y(t)\| \\
&+\frac{4 q b^{\mu} M N_{1}}{\Gamma(\mu+1)}\left[1+b L L_{1}\right]\|x(t)-y(t)\|+\frac{4 m M b^{(v-1)(1-\mu)}}{\Gamma(v(1-\mu)+\mu}\|x(t)-y(t)\| \\
& \leq\left\{\frac{4 q b^{\mu} M N_{1}}{\Gamma(\mu+1)}\left[1+b L L_{1}\right]+\frac{4 m M b^{(v-1)(1-\mu)}}{\Gamma(v(1-\mu)+\mu)}\right\}\left\{1+\frac{M\|B\|\left\|W^{-1}\right\| b^{\mu}}{\Gamma(\mu+1)}\right\}\|x(t)-y(t)\| \\
& \leq \chi \| x(x(t)-y(t) \| .
\end{aligned}
$$

Therefore,

$$
\sup _{t \in J} t^{(1-v)(1-\mu)}\left\|\left(\Phi^{*} x_{1}\right)(t)-\left(\Phi^{*} x_{2}\right)(t)\right\| \leq \chi_{2} \sup _{t \in J} t^{(1-v)(1-\mu)}\left\|x_{1}(t)-x_{2}(t)\right\| .
$$

This implies that

$$
\left\|\Phi^{*} x_{1}-\Phi^{*} x_{2}\right\|_{Y} \leq \chi_{2}\left\|x_{1}-x_{2}\right\|_{Y}
$$


Then $\Phi^{*}$ is a contraction mapping, and hence there exists a unique fixed point $x \in B_{r}$ such that $\Phi^{*} x(t)=x(t)$. Therefore system (3.4) has a mild solution satisfying $x(b)=x_{1}$. Thus, system (3.4) is controllable on $J$.

\section{Existence and approximate controllability}

First, we study existence and uniqueness for Sobolev-type neutral Hilfer fractional differential equation with impulsive condition in the following form:

$$
\left\{\begin{array}{l}
D_{0+}^{v, \mu}\left[Z x(t)+G\left(t, x\left(\gamma_{1}(t)\right)\right]\right. \\
\quad=A x(t)+f\left(t, x\left(\gamma_{2}(t)\right), \int_{0}^{t} h(t, s) g\left(s, x\left(\gamma_{3}(s)\right)\right) d s\right), \quad t \in J=(0, b], t \neq t_{k}, \\
\left.\Delta x\right|_{t=t_{k}}=I_{k}\left(x\left(t_{k}^{-}\right)\right), \quad k=1,2, \ldots, m \\
I_{0+}^{(1-v)(1-\mu)} x(0)=x_{0},
\end{array}\right.
$$

where the delay $\gamma_{i}(t): J \rightarrow J, i=1,2,3$, are continuous functions, the state $x(\cdot)$ takes values in the Banach space $E$, the symbols $A$ and $Z$ are linear operators on $E$.

The operators $A: D(A) \subset E \rightarrow E$ and $Z: D(Z) \subset E \rightarrow E$ satisfy the following conditions:

(H6) $A$ and $Z$ are closed linear operators.

$(H 7) D(Z) \subset D(A)$ and $Z$ is bijective.

$(H 8) Z^{-1}: E \rightarrow D(Z)$ is continuous.

Here, (H6) and $(H 7)$ together with the closed graph theorem imply the boundedness of the linear operator $A Z^{-1}: E \rightarrow E$.

(H9) For each $t \in J$ and for $\lambda \in \rho\left(A Z^{-1}\right)$, the resolvent of $A Z^{-1}$, the resolvent $R\left(\lambda, A Z^{-1}\right)$ is compact operator.

Lemma 4.1 (see [26]) Let $T(t)$ be a uniformly continuous semigroup. If the resolvent set $R(\lambda, A)$ of $A$ is compact for every $\lambda \in \rho(A)$, then $T(t)$ is a compact semigroup.

From the above fact, $A Z^{-1}$ generates a compact semigroup $\{S(t), t>0\}$ in $E$, which means that there exists $M>1$ such that $\sup _{t \in J}\|S(t)\| \leq M$.

We suppose that $0 \in \rho\left(A Z^{-1}\right)$, the resolvent set of $A Z^{-1}$, and $\|S(t)\| \leq M$ for some constant $M \geq 1$ and every $t>0$. We define the fractional power $\left(A Z^{-1}\right)^{-\gamma}$ by

$$
\left(A Z^{-1}\right)^{-\gamma}=\frac{1}{\Gamma(\gamma)} \int_{0}^{\infty} t^{\gamma-1} S(t) d t, \quad \gamma>0 .
$$

For $\gamma \in(0,1],\left(A Z^{-1}\right)^{\gamma}$ is a closed linear operator on its domain $D\left(\left(A Z^{-1}\right)^{\gamma}\right)$. Furthermore, the subspace $D\left(\left(A Z^{-1}\right)^{\gamma}\right)$ is dense in $E$. We will introduce the following basic properties of $\left(A Z^{-1}\right)^{\gamma}$.

Theorem 4.1 (see [27]) (1) Let $0<\gamma \leq 1$, then $E_{\gamma}:=D\left(\left(A Z^{-1}\right)^{\gamma}\right)$ is a Banach space with the norm $\|x\|_{\gamma}=\left\|\left(A Z^{-1}\right)^{\gamma} x\right\|, x \in E_{\gamma}$.

(2) If $0<\beta<\gamma \leq 1$, then $D\left(\left(A Z^{-1}\right)^{\gamma}\right) \hookrightarrow D\left(\left(A Z^{-1}\right)^{\beta}\right)$ and the embedding is compact whenever the resolvent operator of $\left(A Z^{-1}\right)$ is compact.

(3) For every $0<\gamma \leq 1$, there exists a positive constant $C_{\gamma}$ such that

$$
\left\|\left(A Z^{-1}\right)^{\gamma} S(t)\right\| \leq \frac{C_{\gamma}}{t^{\gamma}}, \quad 0<t \leq b
$$


Lemma 4.2 For any $x \in E, \beta \in(0,1)$ and $\delta \in(0,1]$, we have

$$
\left(A Z^{-1}\right) T_{\mu}(t) x=\left(A Z^{-1}\right)^{1-\beta} T_{\mu}(t)\left(A Z^{-1}\right)^{\beta} x, \quad 0<t \leq b
$$

and

$$
\left\|\left(A Z^{-1}\right)^{\delta} T_{\mu}(t) x\right\| \leq \frac{\mu C_{\delta} \Gamma(2-\delta)}{t^{\delta \mu} \Gamma(1+\mu(1-\delta))}\|x\|, \quad 0<t \leq b
$$

Definition 4.1 We say that $x \in Y$ is a mild solution of system (4.1) if the function $A Z^{-1} P_{\mu}(t-s) G\left(s, x\left(\gamma_{1}(s)\right), s \in(0, b)\right.$ is integrable on $(0, b)$ and the following integral equation is verified:

$$
\begin{aligned}
x(t)= & Z^{-1} S_{v, \mu}(t)[Z x(0)+G(0, x(0))]-Z^{-1} G\left(t, x\left(\gamma_{1}(t)\right)\right. \\
& +\int_{0}^{t} Z^{-1} A Z^{-1} P_{\mu}(t-s) G\left(s, x\left(\gamma_{1}(s)\right) d s\right. \\
& +\int_{0}^{t} Z^{-1} P_{\mu}(t-s) f\left(s, x\left(\gamma_{2}(s)\right), \int_{0}^{s} h(s, \tau) g\left(\tau, x\left(\gamma_{3}(\tau)\right)\right) d \tau\right) d s \\
& +Z^{-1} \sum_{0<t_{k}<t} S_{v, \mu}\left(t-t_{k}\right) I_{k}\left(x\left(t_{k}^{-}\right)\right), \quad t \in J .
\end{aligned}
$$

To establish the results, we need the following assumptions.

(H10) (i) $G: J \times E \rightarrow E$ is continuous and there exist constants $K_{1}>0$ and $K_{2}>0$ such that, for all $v_{1}, v_{2} \in B_{r}$, we have

$$
\begin{aligned}
& \left\|\left(A Z^{-1}\right)^{\beta} G\left(t, v_{1}\right)-\left(A Z^{-1}\right)^{\beta} G\left(t, v_{2}\right)\right\| \leq K_{1}\left\|v_{1}-v_{2}\right\|_{Y}, \\
& K_{2}=\left\|\left(A Z^{-1}\right)^{\beta} G(t, 0)\right\|, \quad t \in J .
\end{aligned}
$$

(ii) There exists a constant $q$ such that, for all $x_{1}, x_{2} \in E$,

$$
\left\|x_{1}\left(\gamma_{i}(t)\right)-x_{2}\left(\gamma_{i}(t)\right)\right\| \leq q\left\|x_{1}(t)-x_{2}(t)\right\| \quad \text { for } i=1,2,3 \text {. }
$$

(H11) There exists a constant $r>0$ such that

$$
\begin{aligned}
5 \| & Z^{-1} \|\left\{\frac{M \varrho_{1}}{\Gamma(v(1-\mu)+\mu)}\right. \\
& \left.+\| b^{(1-\nu)(1-\mu)}\left[M_{0}+\frac{C_{1-\beta} b^{\beta \mu} \Gamma(1+\beta)}{\beta \Gamma(1+\mu \beta)}\right] \varrho_{2}+\frac{M b^{1-\nu(1-\mu)} \varrho_{3}}{\mu \Gamma(\mu)}\right\} \leq r
\end{aligned}
$$

and

$$
\begin{gathered}
6\left\|Z^{-1}\right\|\left[1+\varrho_{4}\right]\left\{\frac{M \varrho_{1}}{\Gamma(\nu(1-\mu)+\mu)}+\| b^{(1-v)(1-\mu)}\left[M_{0}+\frac{C_{1-\beta} b^{\beta \mu} \Gamma(1+\beta)}{\beta \Gamma(1+\mu \beta)}\right] \varrho_{2}\right. \\
\left.+\frac{M b^{1-\nu(1-\mu)} \varrho_{3}}{\mu \Gamma(\mu)}\right\}+6 b^{(1-v)(1-\mu)}\|\bar{h}\| \varrho_{4} \leq r,
\end{gathered}
$$


where

$$
\begin{aligned}
& \varrho_{1}=\left(\|Z\|+M_{0} K_{1}\right)\left\|x_{0}\right\|+M_{0} K_{2}+m\left(L_{3} r+L_{4}\right), \quad \varrho_{2}=\left(K_{1} r+K_{2}\right), \\
& \varrho_{3}=N_{1}\left(r+b L\left(L_{1} r+L_{2}\right)\right)+N_{2}, \\
& \varrho_{4}=\frac{M^{2} b^{\mu}\left\|Z^{-1}\right\|\|B\|\left\|B^{*}\right\|}{\lambda \mu \Gamma^{2}(\mu)}, \quad M_{0}=\left\|\left(A Z^{-1}\right)^{-\beta}\right\| .
\end{aligned}
$$

Theorem 4.2 If hypotheses (H1)-(H2) and (H6)-(H11) are satisfied, system (4.1) has a unique mild solution on $J$ provided that

$$
\chi_{3}:=5 q\left\|Z^{-1}\right\|\left\{M_{0} K_{1}+\frac{C_{1-\beta} b^{\beta \mu} K_{1} \Gamma(1+\beta)}{\Gamma(1+\mu \beta)}+\frac{M b^{\mu} N_{1}}{\mu \Gamma(\mu)}\left(1+b L L_{1}\right)+\frac{m M b^{(\nu-1)(1-\mu)}}{\Gamma(\nu(1-\mu)+\mu)}\right\}
$$

$<1$.

Proof Consider the operator $\Psi$ on $Y$ defined as follows:

$$
\begin{aligned}
(\Psi x)(t)= & Z^{-1} S_{v, \mu}(t)[Z x(0)+G(0, x(0))]-Z^{-1} G\left(t, x\left(\gamma_{1}(t)\right)\right. \\
& +\int_{0}^{t} Z^{-1} A Z^{-1} P_{\mu}(t-s) G\left(s, x\left(\gamma_{1}(s)\right) d s\right. \\
& +\int_{0}^{t} Z^{-1} P_{\mu}(t-s) f\left(s, x\left(\gamma_{2}(s)\right), \int_{0}^{s} h(s, \tau) g\left(\tau, x\left(\gamma_{3}(\tau)\right)\right) d \tau\right) d s \\
& +Z^{-1} \sum_{0<t_{k}<t} S_{v, \mu}\left(t-t_{k}\right) I_{k}\left(x\left(t_{k}^{-}\right)\right) .
\end{aligned}
$$

It will be shown that the operator $\Psi$ has a fixed point. This fixed point is then a mild solution of system (4.1). First we show that $\Psi$ maps $B_{r}$ into itself. For $x \in B_{r}$,

$$
\begin{aligned}
\|\Psi x\|_{Y}= & \sup _{t \in J} t^{(1-v)(1-\mu)}\|(\Psi x)(t)\| \\
\leq & 5 \sup _{t \in J} t^{(1-v)(1-\mu)}\left\{\left\|Z^{-1} S_{v, \mu}(t)[Z x(0)+G(0, x(0))]\right\|+\| Z^{-1} G\left(t, x\left(\gamma_{1}(t)\right) \|\right.\right. \\
& +\int_{0}^{t} \| Z^{-1} A Z^{-1} P_{\mu}(t-s) G\left(s, x\left(\gamma_{1}(s)\right) \| d s\right. \\
& +\int_{0}^{t}\left\|Z^{-1} P_{\mu}(t-s)\right\|\left\|f\left(s, x\left(\gamma_{1}(s)\right), \int_{0}^{s} h(s, \tau) g\left(\tau, x\left(\gamma_{2}(\tau)\right)\right) d \tau\right)\right\| d s \\
& \left.+\sum_{0<t_{k}<t}\left\|Z^{-1} S_{v, \mu}\left(t-t_{k}\right) I_{k}\left(x\left(t_{k}^{-}\right)\right)\right\|\right\} \\
\leq & \frac{5 M\left\|Z^{-1}\right\|}{\Gamma(v(1-\mu)+\mu)}\left[\|Z\|\left\|x_{0}\right\|+M_{0}\left(K_{1}\left\|x_{0}\right\|+K_{2}\right)+m\left(L_{3} r+L_{4}\right)\right] \\
& +5 M_{0} b^{(1-v)(1-\mu)}\left\|Z^{-1}\right\|\left[K_{1} r+K_{2}\right] \\
& +\frac{5\left\|Z^{-1}\right\| C_{1-\beta} b^{\beta \mu+(1-v)(1-\mu)} \Gamma(1+\beta)}{\beta \Gamma(1+\mu \beta)}\left[K_{1} r+K_{2}\right] \\
& +\frac{5\left\|Z^{-1}\right\| M b^{1-\nu(1-\mu)}}{\mu \Gamma(\mu)}\left(N_{1}\left(r+b L\left(L_{1} r+L_{2}\right)\right)+N_{2}\right)
\end{aligned}
$$




$$
\begin{aligned}
= & 5\left\|Z^{-1}\right\|\left\{\frac{M \varrho_{1}}{\Gamma(v(1-\mu)+\mu)}+b^{(1-v)(1-\mu)}\left[M_{0}+\frac{C_{1-\beta} b^{\beta \mu} \Gamma(1+\beta)}{\beta \Gamma(1+\mu \beta)}\right] \varrho_{2}\right. \\
& \left.+\frac{M b^{1-\nu(1-\mu)} \varrho_{3}}{\mu \Gamma(\mu)}\right\} \\
\leq & r .
\end{aligned}
$$

Thus $\Psi$ maps $B_{r}$ into itself.

We show that $(\Psi x)(t)$ is continuous on $J$ for any $x \in B_{r}$. Let $0<t \leq b$ and $\epsilon>0$ be sufficiently small, then

$$
\begin{aligned}
&\|(\Psi x)(\cdot+\epsilon)-(\Psi x)(\cdot)\|_{Y} \\
&=\sup _{t \in J} t^{(1-v)(1-\mu)}\|(\Psi x)(t+\epsilon)-(\Psi x)(t)\| \\
& \leq 5\left\|Z^{-1}\right\| \sup _{t \in J} t^{(1-v)(1-\mu)}\left\|\left(S_{v, \mu}(t+\epsilon)-S_{v, \mu}(t)\right)\left(Z x_{0}+G(0, x(0))\right)\right\| \\
&+5\left\|Z^{-1}\right\| \sup _{t \in J} t^{(1-v)(1-\mu)}\left\|G\left(t+\epsilon, x\left(\gamma_{1}(t+\epsilon)\right)\right)-G\left(t, x\left(\gamma_{1}(t)\right)\right)\right\| \\
&+5\left\|Z^{-1}\right\| \sup _{t \in J} t^{(1-v)(1-\mu)} \| \int_{0}^{t+\epsilon} A Z^{-1} P_{\mu}(t+\epsilon-s) G\left(s+\epsilon, x\left(\gamma_{1}(s+\epsilon)\right)\right) d s \\
&-\int_{0}^{t} A Z^{-1} P_{\mu}(t-s) G\left(s, x\left(\gamma_{1}(s)\right)\right) d s \| \\
&+5\left\|Z^{-1}\right\| \sup _{t \in J} t^{(1-v)(1-\mu)} \| \int_{0}^{t+\epsilon} P_{\mu}(t+\epsilon-s) f\left(s, x\left(\gamma_{2}(s)\right),\right. \\
&\left.\int_{0}^{s} h(s, \tau) g\left(\tau, x\left(\gamma_{3}(\tau)\right)\right) d \tau\right) d s \\
&-\int_{0}^{t} P_{\mu}(t-s) f\left(s, x\left(\gamma_{2}(s)\right), \int_{0}^{s} h(s, \tau) g\left(\tau, x\left(\gamma_{3}(\tau)\right)\right) d \tau\right) d s \| \\
&+5\left\|Z^{-1}\right\| \sup _{t \in J} t^{(1-v)(1-\mu)} \| \sum_{0<t_{k}<t+\epsilon} S_{v, \mu}\left(t+\epsilon-t_{k}\right) I_{k}\left(x\left(t_{k}^{-}\right)\right) \\
&-\sum_{0<t_{k}<t} S_{v, \mu}\left(t-t_{k}\right) I_{k}\left(x\left(t_{k}^{-}\right)\right) \| .
\end{aligned}
$$

Clearly, the right-hand side of (4.3) tends to zero as $\epsilon \rightarrow 0$. Hence, $(\Psi x)(t)$ is continuous on $J$.

Next, for $x_{1}, x_{2} \in B_{r}$, we obtain

$$
\begin{aligned}
& \left\|\left(\Psi x_{1}\right)(t)-\left(\Psi x_{2}\right)(t)\right\| \\
& \leq 5\left\|Z^{-1}\right\|\left\{\left\|\left(A Z^{-1}\right)^{-\beta}\right\|\left\|\left(A Z^{-1}\right)^{\beta} G\left(t, x_{1}\left(\gamma_{1}(t)\right)\right)-\left(A Z^{-1}\right)^{\beta} G\left(t, x_{2}\left(\gamma_{1}(t)\right)\right)\right\|\right. \\
& \quad+\int_{0}^{t}(t-s)^{\mu-1}\left\|\left(A Z^{-1}\right)^{1-\beta} T_{\mu}(t-s)\right\| \|\left(A Z^{-1}\right)^{\beta} G\left(s, x_{1}\left(\gamma_{1}(s)\right)\right) \\
& \quad-\left(A Z^{-1}\right)^{\beta} G\left(s, x_{2}\left(\gamma_{1}(s)\right)\right) \| d s \\
& \quad+\int_{0}^{t}\left\|P_{\mu}(t-s)\right\| \| f\left(s, x_{1}\left(\gamma_{1}(s)\right), \int_{0}^{s} h(s, \tau) g\left(\tau, x_{1}\left(\gamma_{2}(\tau)\right)\right) d \tau\right)
\end{aligned}
$$




$$
\begin{aligned}
& -f\left(s, x_{2}\left(\gamma_{1}(s)\right), \int_{0}^{s} h(s, \tau) g\left(\tau, x_{2}\left(\gamma_{2}(\tau)\right)\right) d \tau\right) \| d s \\
& \left.+\sum_{0<t_{k}<t}\left\|S_{v, \mu}\left(t-t_{k}\right)\right\|\left\|I_{k}\left(x_{1}\left(t_{k}^{-}\right)\right)-I_{k}\left(x_{2}\left(t_{k}^{-}\right)\right)\right\|\right\} \\
\leq & 5 q\left\|Z^{-1}\right\|\left\{M_{0} K_{1}+\frac{C_{1-\beta} b^{\beta \mu} K_{1} \Gamma(1+\beta)}{\Gamma(1+\mu \beta)}\right. \\
& \left.+\frac{M b^{\mu} N_{1}}{\mu \Gamma(\mu)}\left(1+b L L_{1}\right)+\frac{m M b^{(v-1)(1-\mu)}}{\Gamma(v(1-\mu)+\mu)}\right\}\left\|x_{1}(t)-x_{2}(t)\right\| \\
\leq & \chi_{3}\left\|x_{1}(t)-x_{2}(t)\right\| .
\end{aligned}
$$

Therefore,

$$
\sup _{t \in J} t^{(1-v)(1-\mu)}\left\|\left(\Psi x_{1}\right)(t)-\left(\Psi x_{2}\right)(t)\right\| \leq \chi_{3} \sup _{t \in J} t^{(1-v)(1-\mu)}\left\|x_{1}(t)-x_{2}(t)\right\| .
$$

This implies that

$$
\left\|\Psi x_{1}-\Psi x_{2}\right\|_{Y} \leq \chi_{3}\left\|x_{1}-x_{2}\right\|_{Y}
$$

Then, $\Psi$ is a contraction mapping on $B_{r}$. From the Banach fixed point theorem, $\Psi$ has a unique fixed point $x(t)$ on $J$. Therefore system (4.1) has a unique mild solution on $J$.

Second, we will study the approximate controllability for Sobolev-type neutral Hilfer fractional differential equation with impulsive condition of the form

$$
\left\{\begin{array}{l}
D_{0+}^{v, \mu}\left[Z x(t)+G\left(t, x\left(\gamma_{1}(t)\right)\right]\right. \\
=A x(t)+B u(t)+f\left(t, x\left(\gamma_{2}(t)\right), \int_{0}^{t} h(t, s) g\left(s, x\left(\gamma_{3}(s)\right)\right) d s\right), \\
\quad t \in J=(0, b], t \neq t_{k}, \\
\left.\Delta x\right|_{t=t_{k}}=I_{k}\left(x\left(t_{k}^{-}\right)\right), \quad k=1,2, \ldots, m \\
I_{0+}^{(1-v)(1-\mu)} x(0)=x_{0},
\end{array}\right.
$$

where the control function $u(\cdot)$ is given in $L^{2}(J, U)$, the Banach space of admissible control functions with $U$ a Banach space. The symbol $B$ stands for a bounded linear from $U$ into $E$.

Definition 4.2 We say that $x \in Y$ is a mild solution of system (4.4) if the function $A Z^{-1} P_{\mu}(t-s) G\left(s, x\left(\gamma_{1}(s)\right), s \in(0, b)\right.$ is integrable on $(0, b)$ and the following integral equation is verified:

$$
\begin{aligned}
x(t)= & Z^{-1} S_{v, \mu}(t)[Z x(0)+G(0, x(0))]-Z^{-1} G\left(t, x\left(\gamma_{1}(t)\right)\right. \\
& +\int_{0}^{t} Z^{-1} A Z^{-1} P_{\mu}(t-s) G\left(s, x\left(\gamma_{1}(s)\right) d s+\int_{0}^{t} Z^{-1} P_{\mu}(t-s) B u(s) d s\right. \\
& +\int_{0}^{t} Z^{-1} P_{\mu}(t-s) f\left(s, x\left(\gamma_{2}(s)\right), \int_{0}^{s} h(s, \tau) g\left(\tau, x\left(\gamma_{3}(\tau)\right)\right) d \tau\right) d s \\
& +Z^{-1} \sum_{0<t_{k}<t} S_{v, \mu}\left(t-t_{k}\right) I_{k}\left(x\left(t_{k}^{-}\right)\right), \quad t \in J .
\end{aligned}
$$


In order to study the approximate controllability for system (4.4), we introduce the following Sobolev-type linear fractional differential system:

$$
\left\{\begin{array}{l}
D_{0+}^{v, \mu} Z x(t)=A x(t)+B u(t), \quad t \in J \\
I_{0+}^{(1-v)(1-\mu)} x(0)=x_{0} .
\end{array}\right.
$$

It is convenient at this point to introduce the operators associated with (4.6) as follows:

$$
\begin{aligned}
& \Gamma_{0}^{b}=\int_{0}^{b}(b-s)^{\mu-1} T_{\mu}(b-s) B B^{*} T_{\mu}^{*}(b-s) d s, \\
& R\left(\lambda, \Gamma_{0}^{b}\right)=\left(\lambda I+\Gamma_{0}^{b}\right)^{-1}, \quad \lambda>0 .
\end{aligned}
$$

Let $x\left(b ; x_{0}, u\right)$ be the state value of $(4.4)$ at terminal state $b$, corresponding to the control $u$ and the initial value $x_{0}$. Denote by $R\left(b, x_{0}\right)=\left\{x\left(b ; x_{0}, u\right): u \in L^{2}(J, U)\right\}$ the reachable set of system (4.4) at terminal time $b$, its closure in $X$ is denoted by $\overline{R\left(b, x_{0}\right)}$.

Definition 4.3 System (4.4) is said to be approximately controllable on the interval $J$ if $\overline{R\left(b, x_{0}\right)}=E$.

Lemma 4.3 (see [28]) The linear system (4.6) is approximate controllable on $J$ if and only if the operator $\lambda R\left(\lambda, \Gamma_{0}^{b}\right)=\lambda\left(\lambda I+\Gamma_{0}^{b}\right)^{-1} \rightarrow 0$ as $\lambda \rightarrow 0^{+}$in the strong operator topology.

We formulate sufficient conditions for the approximate controllability of system (4.4). For this purpose, we first prove the existence of a mild solution for system (4.4). Second we prove that system (4.4) is approximately controllable under certain assumptions.

Theorem 4.3 If hypotheses $(H 1)-(H 2)$ and (H6)-(H11) are satisfied, then system (4.4) has a mild solution on $J$ provided that

$$
\begin{aligned}
\chi_{4}:= & 6 q\left\|Z^{-1}\right\|\left\{M_{0} K_{1}+\frac{C_{1-\beta} b^{\beta \mu} K_{1} \Gamma(1+\beta)}{\Gamma(1+\mu \beta)}+\frac{M b^{\mu} N_{1}}{\mu \Gamma(\mu)}\left(1+b L L_{1}\right)+\frac{m M b^{(\nu-1)(1-\mu)}}{\Gamma(\nu(1-\mu)+\mu)}\right\} \\
& \times\left\{1+\frac{\left\|Z^{-1}\right\| M^{2}\|B\|\left\|B^{*}\right\| b^{\mu}}{\lambda \mu \Gamma^{2}(\mu)}\right\}<1 .
\end{aligned}
$$

Proof Consider the operator $\Psi^{*}$ on $Y$ defined as follows:

$$
\begin{aligned}
\left(\Psi^{*} x\right)(t)= & Z^{-1} S_{v, \mu}(t)[Z x(0)+G(0, x(0))]-Z^{-1} G\left(t, x\left(\gamma_{1}(t)\right)\right. \\
& +\int_{0}^{t} Z^{-1} A Z^{-1} P_{\mu}(t-s) G\left(s, x\left(\gamma_{1}(s)\right) d s\right. \\
& +\int_{0}^{t} Z^{-1} P_{\mu}(t-s) B u(s) d s \\
& +\int_{0}^{t} Z^{-1} P_{\mu}(t-s) f\left(s, x\left(\gamma_{2}(s)\right), \int_{0}^{s} h(s, \tau) g\left(\tau, x\left(\gamma_{3}(\tau)\right)\right) d \tau\right) d s \\
& +Z^{-1} \sum_{0<t_{k}<t} S_{v, \mu}\left(t-t_{k}\right) I_{k}\left(x\left(t_{k}^{-}\right)\right),
\end{aligned}
$$


where

$$
\begin{aligned}
u(t)= & B^{*} T_{\mu}^{*}(b-t)\left(\lambda I+\Gamma_{0}^{b}\right)^{-1}\left\{\bar{h}-Z^{-1} S_{v, \mu}(b)[Z x(0)+G(0, x(0))]+Z^{-1} G\left(b, x\left(\gamma_{1}(b)\right)\right.\right. \\
& -\int_{0}^{b} Z^{-1} A Z^{-1} P_{\mu}(b-s) G\left(s, x\left(\gamma_{1}(s)\right) d s-Z^{-1} \sum_{0<t_{k}<b} S_{v, \mu}\left(b-t_{k}\right) I_{k}\left(x\left(t_{k}^{-}\right)\right)\right. \\
& \left.-\int_{0}^{b} Z^{-1} P_{\mu}(b-s) f\left(s, x\left(\gamma_{2}(s)\right), \int_{0}^{s} h(s, \tau) g\left(\tau, x\left(\gamma_{3}(\tau)\right)\right) d \tau\right) d s\right\} .
\end{aligned}
$$

It will be shown that the operator $\Psi^{*}$ has a fixed point. This fixed point is then a mild solution of system (4.4). We show that $\Psi^{*}$ maps $B_{r}$ into itself. For $x \in B_{r}$,

$$
\begin{aligned}
\left\|\Psi^{*} x\right\|_{Y}= & \sup _{t \in J} t^{(1-v)(1-\mu)}\left\|\left(\Psi^{*} x\right)(t)\right\| \\
\leq & 6 \sup _{t \in J} t^{(1-v)(1-\mu)}\left\{\left\|Z^{-1} S_{v, \mu}(t)[Z x(0)+G(0, x(0))]\right\|+\| Z^{-1} G\left(t, x\left(\gamma_{1}(t)\right) \|\right.\right. \\
& +\int_{0}^{t} \| Z^{-1} A Z^{-1} P_{\mu}(t-s) G\left(s, x\left(\gamma_{1}(s)\right) \| d s\right. \\
& +\int_{0}^{t}\left\|Z^{-1} P_{\mu}(t-s)\right\|\|B\|\|u(s)\| d s \\
& +\int_{0}^{t}\left\|Z^{-1} P_{\mu}(t-s)\right\| f\left(s, x\left(\gamma_{1}(s)\right), \int_{0}^{s} h(s, \tau) g\left(\tau, x\left(\gamma_{2}(\tau)\right)\right) d \tau\right) \| d s \\
& \left.+\sum_{0<t_{k}<t}\left\|Z^{-1} S_{v, \mu}\left(t-t_{k}\right) I_{k}\left(x\left(t_{k}^{-}\right)\right)\right\|\right\} \\
\leq & \frac{6 M\left\|Z^{-1}\right\|}{\Gamma(v(1-\mu)+\mu)}\left[\|Z\|\left\|x_{0}\right\|+M_{0}\left(K_{1}\left\|x_{0}\right\|+K_{2}\right)+m\left(L_{3} r+L_{4}\right)\right] \\
& +6 M_{0} b^{(1-v)(1-\mu)}\left\|Z^{-1}\right\|\left[K_{1} r+K_{2}\right] \\
& +\frac{6\left\|Z^{-1}\right\| C_{1-\beta} b^{\beta \mu+(1-v)(1-\mu)} \Gamma(1+\beta)}{\beta \Gamma(1+\mu \beta)}\left[K_{1} r+K_{2}\right]+\frac{6 M^{2} b^{\mu}\left\|Z^{-1}\right\|\|B\|\left\|B^{*}\right\|}{\lambda \mu \Gamma^{2}(\mu)} \\
& \times\left\{b^{(1-v)(1-\mu)}\|\bar{h}\|\right. \\
& +\frac{6\left\|Z^{-1}\right\| M b^{1-v(1-\mu)}}{\mu \Gamma(\mu)}\left(N_{1}\left(r+b L\left(L_{1} r+L_{2}\right)\right)+N_{2}\right) \\
& +\frac{M\left\|Z^{-1}\right\|}{\Gamma(v(1-\mu)+\mu)}\left[\|Z\|\left\|x_{0}\right\|+Z_{0}^{-1} \|\left[1+\varrho_{4}\right]\left\{\frac{M \varrho_{1}}{\Gamma(v(1-\mu)}\left[K_{1}\left\|x_{0}\right\|+K_{2}\right)+m\left(L_{3} r+L_{4}\right)\right]\right. \\
& +\left\|Z^{-1}\right\| b^{(1-v)(1-\mu)}\left(K_{1} r+K_{2}\right)\left[M_{0}+\frac{C_{1-\beta} b^{\beta \mu} \Gamma(1+\beta)}{\beta \Gamma(1+\mu \beta)}\right] \\
& \left.\frac{\left\|Z^{-1}\right\| M b^{1-v(1-\mu)}}{\mu \Gamma(\mu)}\left[N_{1}\left(r+b L\left(L_{1} r+L_{2}\right)\right)+N_{2}\right]\right\} \\
& \\
&
\end{aligned}
$$




$$
\begin{aligned}
& +6 b^{(1-v)(1-\mu)}\|\bar{h}\| \varrho_{4} \\
\leq & r .
\end{aligned}
$$

Thus $\Psi^{*}$ maps $B_{r}$ into itself.

Next, for $x_{1}, x_{2} \in B_{r}$, we obtain

$$
\begin{aligned}
& \left\|\left(\Psi^{*} x_{1}\right)(t)-\left(\Psi^{*} x_{2}\right)(t)\right\| \\
& \leq 6\left\|Z^{-1}\right\|\left\{\left\|\left(A Z^{-1}\right)^{-\beta}\right\|\left\|\left(A Z^{-1}\right)^{\beta} G\left(t, x_{1}\left(\gamma_{1}(t)\right)\right)-\left(A Z^{-1}\right)^{\beta} G\left(t, x_{2}\left(\gamma_{1}(t)\right)\right)\right\|\right. \\
& +\int_{0}^{t}(t-s)^{\mu-1}\left\|\left(A Z^{-1}\right)^{1-\beta} T_{\mu}(t-s)\right\| \|\left(A Z^{-1}\right)^{\beta} G\left(s, x_{1}\left(\gamma_{1}(s)\right)\right) \\
& -\left(A Z^{-1}\right)^{\beta} G\left(s, x_{2}\left(\gamma_{1}(s)\right)\right) \| d s \\
& +\int_{0}^{t}\left\|P_{\mu}(t-s)\right\| \| f\left(s, x_{1}\left(\gamma_{1}(s)\right), \int_{0}^{s} h(s, \tau) g\left(\tau, x_{1}\left(\gamma_{2}(\tau)\right)\right) d \tau\right) \\
& -f\left(s, x_{2}\left(\gamma_{1}(s)\right), \int_{0}^{s} h(s, \tau) g\left(\tau, x_{2}\left(\gamma_{2}(\tau)\right)\right) d \tau\right) \| d s \\
& \left.+\sum_{0<t_{k}<t}\left\|S_{v, \mu}\left(t-t_{k}\right)\right\|\left\|I_{k}\left(x_{1}\left(t_{k}^{-}\right)\right)-I_{k}\left(x_{2}\left(t_{k}^{-}\right)\right)\right\|\right\} \\
& +6\left\|Z^{-1}\right\| \int_{0}^{t}\left\|P_{\mu}(t-s)\right\|\|B\|\left\|B^{*}\right\|\left\|T_{\mu}^{*}(b-s)\right\|\left(\lambda I+\Gamma_{0}^{b}\right)^{-1}\left[\left\|\left(A Z^{-1}\right)^{-\beta}\right\|\right. \\
& \times\left\|\left(A Z^{-1}\right)^{\beta} G\left(b, x_{1}\left(\gamma_{1}(b)\right)\right)-\left(A Z^{-1}\right)^{\beta} G\left(b, x_{2}\left(\gamma_{1}(b)\right)\right)\right\| \\
& +\int_{0}^{b}(b-s)^{\mu-1}\left\|\left(A Z^{-1}\right)^{1-\beta} T_{\mu}(b-s)\right\| \\
& \times\left\|\left(A Z^{-1}\right)^{\beta} G\left(s, x_{1}\left(\gamma_{1}(s)\right)\right)-\left(A Z^{-1}\right)^{\beta} G\left(s, x_{2}\left(\gamma_{1}(s)\right)\right)\right\| d s+\int_{0}^{b}\left\|P_{\mu}(b-\tau)\right\| \\
& \times \| f\left(\tau, x_{1}\left(\gamma_{1}(\tau)\right), \int_{0}^{\tau} h(\tau, \eta) g\left(\eta, x_{1}\left(\gamma_{2}(\eta)\right)\right) d \eta\right) \\
& -f\left(\tau, x_{2}\left(\gamma_{1}(\tau)\right), \int_{0}^{\tau} h(\tau, \eta) g\left(\eta, x_{2}\left(\gamma_{2}(\eta)\right)\right) d \eta\right) \| d \tau \\
& \left.+\sum_{0<t_{k}<b}\left\|S_{v, \mu}\left(b-t_{k}\right)\right\|\left\|I_{k}\left(x_{1}\left(t_{k}^{-}\right)\right)-I_{k}\left(x_{2}\left(t_{k}^{-}\right)\right)\right\|\right] d s \\
& \leq 6 q\left\|Z^{-1}\right\|\left\{M_{0} K_{1}+\frac{C_{1-\beta} b^{\beta \mu} K_{1} \Gamma(1+\beta)}{\Gamma(1+\mu \beta)}+\frac{M b^{\mu} N_{1}}{\mu \Gamma(\mu)}\left(1+b L L_{1}\right)\right. \\
& \left.+\frac{m M b^{(\nu-1)(1-\mu)}}{\Gamma(v(1-\mu)+\mu)}\right\} \\
& \times\left\{1+\frac{\left\|Z^{-1}\right\| M^{2}\|B\|\left\|B^{*}\right\| b^{\mu}}{\lambda \mu \Gamma^{2}(\mu)}\right\}\left\|x_{1}(t)-x_{2}(t)\right\| \\
& \leq \chi_{4}\left\|x_{1}(t)-x_{2}(t)\right\| \text {. }
\end{aligned}
$$


Therefore,

$$
\sup _{t \in J} t^{(1-\nu)(1-\mu)}\left\|\left(\Psi^{*} x_{1}\right)(t)-\left(\Psi^{*} x_{2}\right)(t)\right\| \leq \chi_{4} \sup _{t \in J} t^{(1-v)(1-\mu)}\left\|x_{1}(t)-x_{2}(t)\right\| .
$$

This implies that

$$
\left\|\Psi^{*} x_{1}-\Psi^{*} x_{2}\right\|_{Y} \leq \chi_{4}\left\|x_{1}-x_{2}\right\|_{Y}
$$

Then $\Psi^{*}$ is a contraction mapping and hence there exists a unique fixed point $x \in B_{r}$ such that $\Psi^{*} x(t)=x(t)$. Hence, any fixed point of $\Psi^{*}$ is a mild solution of (4.4) on $J$.

Theorem 4.4 Assume that hypotheses (H1)-(H2) and (H6)-(H11) hold. Further, if the functions $f: J \times E \times E \rightarrow E, G: J \times E \rightarrow E$ are uniformly bounded and $\{S(t), t>0\}$ is compact, then system (4.4) is approximately controllable on J.

Proof Let $x^{\lambda}(\cdot)$ be a fixed point of $\Psi^{*}$ in $B_{r}$. Any fixed point of $\Psi^{*}$ is a mild solution of (4.4) on $J$ under the control

$$
u^{\lambda}(t)=B^{*} T_{\mu}^{*}(b-t) R\left(\lambda, \Gamma_{0}^{b}\right) p\left(x^{\lambda}\right)
$$

where

$$
\begin{aligned}
p\left(x^{\lambda}\right)= & h-Z^{-1} S_{v, \mu}(b)[Z x(0)+G(0, x(0))]+Z^{-1} G\left(b, x^{\lambda}\left(\gamma_{1}(b)\right)\right. \\
& -\int_{0}^{b} Z^{-1} A Z^{-1} P_{\mu}(b-s) G\left(s, x^{\lambda}\left(\gamma_{1}(s)\right) d s\right. \\
& -Z^{-1} \sum_{0<t_{k}<b} S_{v, \mu}\left(b-t_{k}\right) I_{k}\left(x^{\lambda}\left(t_{k}^{-}\right)\right) \\
& -\int_{0}^{b} Z^{-1} P_{\mu}(b-s) f\left(s, x^{\lambda}\left(\gamma_{2}(s)\right), \int_{0}^{s} h(s, \tau) g\left(\tau, x^{\lambda}\left(\gamma_{3}(\tau)\right)\right) d \tau\right) d s,
\end{aligned}
$$

and satisfies

$$
x^{\lambda}(b)=h-\lambda\left(\lambda I+\Gamma_{0}^{b}\right)^{-1} p\left(x^{\lambda}\right)
$$

It follows from the assumption on $f$ and $G$ that there exists $D>0$ such that

$$
\begin{aligned}
& \left\|f\left(s, x^{\lambda}\left(\gamma_{2}(s)\right), \int_{0}^{s} k(s, \tau) g\left(\tau, x^{\lambda}\left(\gamma_{3}(\tau)\right)\right) d \tau\right)\right\| \leq D, \\
& \left\|G\left(s, x^{\lambda}\left(\gamma_{1}(s)\right)\right)\right\| \leq D .
\end{aligned}
$$

Consequently, the sequence $\left\{G\left(s, x^{\lambda}\left(\gamma_{1}(s)\right)\right), f\left(s, x^{\lambda}\left(\gamma_{2}(s)\right), \int_{0}^{s} k(s, \tau) g\left(\tau, x^{\lambda}\left(\gamma_{3}(\tau)\right)\right) d \tau\right)\right\}$ is bounded in $L^{2}(J, E)$.

Thus there is a subsequence, still denoted by $\left\{G\left(s, x^{\lambda}\left(\gamma_{1}(s)\right)\right), f\left(s, x^{\lambda}\left(\gamma_{2}(s)\right), \int_{0}^{s} k(s, \tau) \times\right.\right.$ $\left.\left.g\left(\tau, x^{\lambda}\left(\gamma_{3}(\tau)\right)\right) d \tau\right)\right\}$, that converges to say $\{G(s), f(s)\}$. On the other hand, by Lemma 4.3, the operator $\lambda\left(\lambda I+\Gamma_{0}^{b}\right)^{-1} \rightarrow 0$ strongly as $\lambda \rightarrow 0^{+}$for all $0<s \leq b$, and, moreover, $\left\|\lambda\left(\lambda I+\Gamma_{0}^{b}\right)^{-1}\right\| \leq 1$. 
Thus, the Lebesgue dominated convergence theorem and the compactness of $P_{\mu}(t)$ yield

$$
\begin{aligned}
\| x^{\lambda}(b) & -h \| \\
\leq & \left\|\lambda\left(\lambda I+\Gamma_{0}^{b}\right)^{-1}\left[h-Z^{-1} S_{v, \mu}(b)(Z x(0)+G(0, x(0)))\right]\right\| \\
& +\| \lambda\left(\lambda I+\Gamma_{0}^{b}\right)^{-1} Z^{-1} G\left(b, x^{\lambda}\left(\gamma_{1}(b)\right) \|\right. \\
& +\int_{0}^{b} \| \lambda\left(\lambda I+\Gamma_{0}^{b}\right)^{-1} Z^{-1} A Z^{-1} P_{\mu}(b-s) G\left(s, x^{\lambda}\left(\gamma_{1}(s)\right) \| d s\right. \\
& +\int_{0}^{b}\left\|\lambda\left(\lambda I+\Gamma_{0}^{b}\right)^{-1} Z^{-1} P_{\mu}(b-s) f\left(s, x^{\lambda}\left(\gamma_{2}(s)\right), \int_{0}^{s} k(s, \tau) g\left(\tau, x^{\lambda}\left(\gamma_{3}(\tau)\right)\right) d \tau\right)\right\| d s \\
& +\left\|\lambda\left(\lambda I+\Gamma_{0}^{b}\right)^{-1}\right\|\left\|Z^{-1} \sum_{0<t_{k}<b} S_{v, \mu}\left(b-t_{k}\right) I_{k}\left(x^{\lambda}\left(t_{k}^{-}\right)\right)\right\| \rightarrow 0, \quad \text { as } \lambda \rightarrow 0^{+} .
\end{aligned}
$$

This gives the approximate controllability of (4.4), the proof is complete.

\section{Application}

Consider the following Sobolev-type Hilfer fractional delay partial differential equation with impulsive condition:

$$
\left\{\begin{array}{l}
D_{0+}^{v, \frac{3}{5}}\left[x(t, y)-x_{y y}(t, y)+\tilde{G}(t, x(t-\rho, y))\right] \\
\quad=\frac{\partial^{2} x(t, y)}{\partial y^{2}}+\chi(t, y)+x(t-\rho, y)+\int_{0}^{t} \sin x(s-\rho, y) d s, \\
\quad 0 \leq y \leq \pi, t \in J=(0, b], t \neq t_{k}, \\
x(t, 0)=x(t, \pi)=0, \quad t \in J, \\
x\left(t_{k}^{+}, y\right)-x\left(t_{k}^{-}, y\right)=I_{k}\left(x\left(t_{k}^{-}, y\right)\right), \quad k=1,2, \ldots, m, \\
I_{0+}^{\frac{2}{5}(1-v)}(x(0, y))=x_{0}(y), \quad 0 \leq y \leq \pi,
\end{array}\right.
$$

where $D_{0+}^{v, \frac{3}{5}}$ is a Hilfer fractional derivative of order $0 \leq v \leq 1, \mu=\frac{3}{5}$.

Let $E=U=L^{2}([0, \pi])$, define the operators $Z: D(Z) \subset E \rightarrow E$ and $A: D(A) \subset E \rightarrow E$ by $Z x=x-x_{y y}$ and $A x=x_{y y}$, where the domains $D(Z)$ and $D(A)$ are given by $\{x \in E$ : $x, x_{y}$ are absolutely continuous, $\left.x_{y y} \in E, x(0)=x(\pi)=0\right\}$.

Then $A$ and $Z$ can be written as

$$
A x=\sum_{n=1}^{\infty} n^{2}\left(x, x_{n}\right) x_{n}, \quad x \in D(A), \quad Z x=\sum_{n=1}^{\infty}\left(1+n^{2}\right)\left(x, x_{n}\right) x_{n}, \quad x \in D(Z) .
$$

Furthermore, for $x \in E$, we have

$$
Z^{-1} x=\sum_{n=1}^{\infty} \frac{1}{1+n^{2}}\left(x, x_{n}\right) x_{n}, \quad A Z^{-1} x=\sum_{n=1}^{\infty} \frac{n^{2}}{1+n^{2}}\left(x, x_{n}\right) x_{n}
$$

It is known that $A Z^{-1}$ is self-adjoint and has the eigenvalues $\lambda_{n}=-n^{2} \pi^{2}, n \in N$, with the corresponding normalized eigenvectors $e_{n}(\xi)=\sqrt{2} \sin (n \pi \xi)$. Furthermore, $A Z^{-1}$ generates a uniformly strongly continuous semigroup of bounded linear operators $S(t), t>0$, on 
$E$ which is given by

$$
S(t) \xi=\sum_{n=1}^{\infty}\left(\xi_{n}, e_{n}\right) e_{n}=\sum_{n=1}^{\infty} 2 e^{-n^{2} \pi^{2} t} \sin (n \pi y) \int_{0}^{1} \sin (n \pi \tau) \xi(\tau) d \tau, \quad \xi \in E
$$

We define the bounded operator $B: U \rightarrow E$ by $B u=\chi(t, y), 0 \leq y \leq \pi, u \in U$.

Also, we define the following functions:

$$
\begin{aligned}
& x(t) y=x(t, y), G\left(t, x\left(\gamma_{1}(t)\right)\right)(y)=\tilde{G}(t, x(t-\rho, y)), \\
& \int_{0}^{t} h(t, s) g\left(s, x\left(\gamma_{3}(s)\right)(y) d s=\int_{0}^{t} \sin x(s-\rho, y) d s,\right. \\
& f\left(t, x\left(\gamma_{2}(t)\right), \int_{0}^{t} h(t, s) g\left(s, x\left(\gamma_{2}(s)\right)\right) d s\right)(y)=x(t-\rho, y)+\int_{0}^{t} \sin x(s-\rho, y) d s,
\end{aligned}
$$

where $h(t, s)=1$. Choose b and other constants such that conditions $(H 1)-(H 2)$ and $(H 6)-$ (H11) are satisfied.

Hence, all the hypotheses of Theorem 4.3 and Theorem 4.4 are satisfied and

$$
\begin{aligned}
\chi_{4}:= & 6 q\left\|Z^{-1}\right\|\left\{M_{0} K_{1}+\frac{C_{1-\beta} b^{\beta \mu} K_{1} \Gamma(1+\beta)}{\Gamma(1+\mu \beta)}+\frac{M b^{\mu} N_{1}}{\mu \Gamma(\mu)}\left(1+b L L_{1}\right)+\frac{m M b^{(\nu-1)(1-\mu)}}{\Gamma(v(1-\mu)+\mu)}\right\} \\
& \times\left\{1+\frac{\left\|Z^{-1}\right\| M^{2}\|B\|\left\|B^{*}\right\| b^{\mu}}{\lambda \mu \Gamma^{2}(\mu)}\right\}<1 .
\end{aligned}
$$

So the Sobolev-type Hilfer fractional delay partial differential equation with impulsive condition (5.1) is approximately controllable on $J$.

\section{Acknowledgements}

The authors would like to thank the referees and the editor for their useful suggestions, which have significantly improved the paper.

\section{Funding}

Not applicable.

\section{Competing interests}

The authors declare that they have no competing interests.

\section{Authors' contributions}

All authors contributed equally to each part of this work. All authors read and approved the final manuscript.

\section{Author details}

${ }^{1}$ Higher Institute of Engineering, El-Shorouk Academy, Cairo, Egypt. ${ }^{2}$ Department of Mathematics, Faculty of Science, Alexandria University, Cairo, Egypt. ${ }^{3}$ Department of Mathematics, Faculty of Science, Al-Azhar University, Cairo, Egypt.

\section{Publisher's Note}

Springer Nature remains neutral with regard to jurisdictional claims in published maps and institutional affiliations.

Received: 7 March 2018 Accepted: 4 June 2018 Published online: 28 June 2018

\section{References}

1. El-Borai, M.M.: Some probability densities and fundamental solutions of fractional evolution equations. Chaos Solitons Fractals 14(3), 433-440 (2002)

2. Zhou, Y., Jiao, F.: Existence of mild solutions for fractional neutral evolution equations. Comput. Math. Appl. 59, 1063-1077 (2010)

3. Kilbas, A.A., Srivastava, H.M., Trujillo, J.J.: Theory and Applications of Fractional Differential Equations. North-Holland Mathematics Studies, vol. 204. Elsevier, Amsterdam (2006) 
4. Wang, J.R., Feckan, M., Zhou, Y.: A survey on impulsive fractional differential equations. Fract. Calc. Appl. Anal. 19(4), 806-831 (2016)

5. Riveros, M.S., Vidal, R.E.: Sharp bounds for fractional one-sided operators. Acta Math. Sin. Engl. Ser. 32(11), 1255-1278 (2016)

6. Abbas, S., Benchohra, M., Lazreg, J.-E., Zhou, Y:: A survey on Hadamard and Hilfer fractional differential equations: analysis and stability. Chaos Solitons Fractals 102, 47-71 (2017)

7. Ahmed, H.M., El-Borai, M.M.: Hilfer fractional stochastic integro-differential equations. Appl. Math. Comput. 331, 182-189 (2018)

8. Benchohra, M., Lazreg, J.E.: Exsitence and Ulam stability for nonlinear implicit fractional differential equations with Hadamard derivative. Stud. Univ. Babeş-Bolyai, Math. 62, 27-38 (2017)

9. Morales-Delgadoa, V.F., Gómez-Aguilarb, J.F., Taneco-Hernandez, M.A.: Analytical solutions of electrical circuits described by fractional conformable derivatives in Liouville-Caputo sense. AEÜ, Int. J. Electron. Commun. 85, 108-117 (2018)

10. Vivek, D., Kanagarajan, K., Elsayed, E.M.: Some existence and stability results for Hilfer-fractional implicit differential equations with nonlocal conditions. Mediterr. J. Math. (2018). https://doi.org/10.1007/s00009-017-1061-0

11. Sakthivel, R., Ganesh, R., Anthoni, S.M.: Approximate controllability of fractional nonlinear differential inclusions. Appl. Math. Comput. 225, 708-717 (2013)

12. Sakthivel, R., Ren, Y: Approximate controllability of fractional differential equations with state-dependent delay. Results Math. 63, 949-963 (2013)

13. Ahmed, H.M.: Controllability for Sobolev type fractional integro-differential systems in a Banach space. Adv. Differ. Equ. 2012, 167 (2012)

14. Ahmed, H.M.: Controllability of impulsive neutral stochastic differential equations with fractional Brownian motion IMA J. Math. Control Inf. (2014)

15. Ahmed, H.M.: Approximate controllability of impulsive neutral stochastic differential equations with fractional Brownian motion in a Hilbert space. Adv. Differ. Equ. 2014, 113 (2014)

16. Debbouche, A., Torres, D.F.M.: Approximate controllability of fractional delay dynamic inclusions with nonlocal control conditions. Appl. Math. Comput. 243, 161-175 (2014)

17. Ahmed, H.M.: Non-linear fractional integro-differential systems with non-local conditions. IMA J. Math. Control Inf. 33 389-399 (2016)

18. Wang, J., Ahmed, H.M.: Null controllability of nonlocal Hilfer fractional stochastic differential equations. Miskolc Math. Notes 18(2), 1073-1083 (2017)

19. Muthukumar, P., Thiagu, K.: Existence of solutions and approximate controllability of fractional nonlocal neutral impulsive stochastic differential equations of order $1<q<2$ with infinite delay and Poisson jumps. J. Dyn. Contro Syst. 23, 213-235 (2017)

20. Yan, Z., Lu, F.: Approximate controllability of a multi-valued fractional impulsive stochastic partial integro-differential equation with infinite delay. Appl. Math. Comput. 292, 425-447 (2017)

21. Wang, J., Feckan, M., Zhou, Y.: Approximate controllability of Sobolev type fractional evolution systems with nonlocal conditions. Evol. Equ. Control Theory 6(3), 471-486 (2017)

22. Miller, K.S., Ross, B.: An Introduction to the Fractional Calculus and Fractional Differential Equations. Wiley, New York (1993)

23. Hilfer, R.: Applications of Fractional Calculus in Physics. World Scientific, Singapore (2000)

24. Hilfer, R.: Experimental evidence for fractional time evolution in glass materials. Chem. Phys. 284, 399-408 (2002)

25. Gu, H., Trujillo, J.J.: Existence of mild solution for evolution equation with Hilfer fractional derivative. Appl. Math. Comput. 257, 344-354 (2015)

26. Curtain, R.F., Zwart, H.: An Introduction to Infinite Dimensional Linear Systems Theory. Springer, New York (1995)

27. Pazy, A.: Semigroups of Linear Operators and Applications to Partial Differential Equations, vol. 44. Springer, New York (1983)

28. Mahmoudov, N.I., Denker, A.: On controllability of linear stochastic systems. Int. J. Control 73, 144-151 (2000)

\section{Submit your manuscript to a SpringerOpen ${ }^{\circ}$ journal and benefit from:}

- Convenient online submission

- Rigorous peer review

- Open access: articles freely available online

- High visibility within the field

Retaining the copyright to your article

Submit your next manuscript at $\gg$ springeropen.com 